\title{
Comportamento Suicidário nos Internos de Psiquiatria em Portugal: Comparação com a Realidade Europeia
}

\author{
Suicidal Behavior among Portuguese Psychiatry Trainees: Comparison with \\ the European Situation
}

\author{
João GAMA MARQUES ${ }^{1,2}$, Alice ROBERTO $\rrbracket^{3}$, Cátia GUERRA ${ }^{4}$, Mariana PINTO DA COSTA ${ }^{5}$, Anja PODLESEK $^{6}$, \\ Julian BEEZHOLD ${ }^{7,8}$, Nikolina JOVANOVIC ${ }^{9}$, Ana MOSCOSO ${ }^{10}$ \\ Acta Med Port 2015 Sep-Oct;28(5):608-612
}

\section{RESUMO}

Introdução: O objectivo deste trabalho foi avaliar a prevalência de ideação suicida e tentativas de suicídio nos médicos internos de psiquiatria de adultos e de psiquiatria da infância e da adolescência em Portugal, e comparar os resultados com a população geral portuguesa e de outros países europeus.

Material e Métodos: Enviou-se um questionário estruturado e anónimo, por e-mail, aos 159 internos de psiquiatria de adultos e de psiquiatria da infância e da adolescência do país, questionando antecedentes pessoais de ideação suicida e tentativas de suícidio, bem como antecedentes familiares de tentativas de suicídio falhadas e consumadas. Estas questões fazem parte do Estudo BoSS (Burnout Syndrome Study) realizado em 21 países. A análise dos dados foi feita através do programa informático SPSS v. 19.

Resultados: Responderam parcialmente ao questionário 62 internos (40,3\%) e 46 (29\%) responderam ao questionário na totalidade, constituindo assim a amostra. O ratio feminino:masculino foi de $2: 1$ e a média de idade de 29 anos. A ideação suicida estava presente na forma passiva em $44 \%$ dos inquiridos e na forma activa em $33 \% ; 4,3 \%$ referiu tentativas de suicídio prévias. Em relação à história familiar, registou-se $22 \%$ de tentativas de suicídio e $13 \%$ de suicídio consumado.

Discussão: Os resultados obtidos são preocupantes e podem estar associados a factores específicos a que esta população está exposta.

Conclusão: É necessária uma investigação mais aprofundada para se compreender melhor este fenómeno, respectivas causas e potenciais modificadores.

Palavras-chave: Burnout Profissional; Europa; Internato e Residência; Médicos; Portugal; Psiquiatria; Stress Psicológico; Suicídio; Tentativa de Suicídio.

\section{ABSTRACT}

Introduction: The aim of this paper was to assess the prevalence of suicide ideation and attempts in Portuguese psychiatry trainees (adult and child and adolescence), and compare the data with the general population and other European countries.

Material and Methods: A structured and anonymous questionnaire was sent by email to 159 portuguese trainees of adult psychiatry and child and adolescence psychiatry with questions about personal history of suicidal ideation and suicide attempts, as well as family history of suicide attempts and completed suicides. This is part of the BoSS Study (Burnout Syndrome Study) performed in 21 countries worldwide. Data was analysed in SPSS v.19.

Results: From the inquired population, 62 trainees $(40,3 \%)$ partially responded, and $46(29 \%)$ were complete responders - these entered the final analysis. There was a ratio of 2:1 (female:male) and a mean age of 29 years. The suicidal ideation was present in passive form in $44 \%$ and in active form in $33 \%$; also, $4.3 \%$ of respondents had previous suicide attempts. In first degree relatives, $22 \%$ had attempted suicide and $13 \%$ completed suicide.

Discussion: The results are worriying and may be associated with some factors to which this population is exposed.

Conclusion: It is necessary further research to better understand this phenomenon, its causes and potential modifiers.

Keywords: Burnout, Professional; Europe; Internship and Residency; Physicians; Psychiatry; Stress, Psychological; Suicide; Suicide, Attempted.

\section{INTRODUÇÃO}

Em Portugal a patologia psiquiátrica apresenta valores superiores aos da maioria dos países europeus. ${ }^{1}$ Relativamenteaosuicídio, apesardasua prevalência parecer ser subestimada, em 2010 registaram-se 1098 óbitos por suicídio, ${ }^{2}$ sendo actualmente a primeira causa de morte

não natural. ${ }^{3}$ Apesar do número de suicídios se ter mantido estável entre 2011-2012, o Instituto Nacional de Emergência Médica verificou nesse período um acréscimo relevante $(41 \%)$ de pedidos de ajuda para evitar potenciais casos suicidas, situação que merece a reflexão da sociedade. ${ }^{4,5}$

1. Clínica de Psicoses Esquizofrénicas. Centro Hospitalar Psiquiátrico de Lisboa. Lisboa. Portugal

2. Clínica Universitária de Psiquiatria e Psicologia Médica. Faculdade de Medicina. Universidade de Lisboa. Lisboa. Portugal.

3. Departamento de Psiquiatria. Hospital Garcia de Orta. Almada. Portugal.

4. Departamento de Psiquiatria. Centro Hospitalar de S. João. Porto. Portugal.

5. Hospital de Magalhães Lemos. Universidade do Porto. Porto. Portugal.

6. Department of Psychology. University of Ljubljana. Ljubljana. Slovenia.

7. Department of Psychiatry. Hellesdon Hospital. Norwich. Norfolk. United Kingdom.

8. University of East Anglia. Norwich. United Kingdom.

9. Psychiatric Clinic. University Hospital Centre and School of Medicine. Zagreb. Croatia.

10. Departamento de Pedopsiquiatra. Hospital de D. Estefânia. Centro Hospitalar de Lisboa Central. Lisboa. Portugal.

$\bowtie$ Autor correspondente: Alice Roberto. alice.roberto@gmail.com

Recebido: 27 de Novembro de 2014 - Aceite: 27 de Julho de 2015 | Copyright @ Ordem dos Médicos 2015 
Estudos recentes apontam para um risco suicidário maior nos médicos do que na população em geral, ${ }^{6,7}$ facto que vem sendo discutido desde a década de 70 do século passado, existindo no entanto ainda significativa controvérsia acerca da frequência destes comportamentos: verificar-se-á de facto o dobro da prevalência da população geral $^{7}$ ou estarão estes resultados inflaccionados por erros metodológicos? ${ }^{8}$

Um dos factores que parece contribuir para essa vulnerabilidade é a ocorrência da síndrome de burnout. A síndrome de burnout caracteriza-se pela presença de exaustão emocional, cinismo ou desapego às exigências profissionais intensas, e de uma percepção negativa do desempenho profissional pessoal. ${ }^{9}$ A síndrome tem sido avaliada em estudantes de medicina, ${ }^{10,11}$ médicos internos e especialistas, ${ }^{12}$ em particular nas especialidades de anestesia, medicina geral e familiar e psiquiatria. ${ }^{13}$ Apesar da síndrome de burnout ser esperada em etapas avançadas da carreira médica, devido ao stress ocupacional prolongado, ele parece ser mais frequente em internos ${ }^{14} \mathrm{e}$ médicos em início de carreira. ${ }^{15}$

Na classe médica, em particular, têm sido descritas taxas de burnout na ordem dos $30 \%$ a $65 \%$ variando consoante a especialidade, sendo que os mais vulneráveis parecem ser os médicos dos cuidados de saúde primários e da medicina de emergência. ${ }^{16}$ Num recente inquérito realizado pela Medscape, a taxa de burnout foi de $38 \%$ em médicos psiquiatras de todas as idades e de $43 \%$ em médicos psiquiatras com menos de 35 anos. ${ }^{17}$ Esta diferença etária alerta-nos para uma maior vulnerabilidade nos médicos em internato ou início de carreira, quando comparados com os colegas mais séniores. De facto burnout e comportamentos suicidários parecem estar relacionados não só durante o internato, mas ainda antes disso, ou seja, logo na faculdade durante o curso de medicina. ${ }^{18}$

O grupo internacional Burnout Syndrome Study (BoSS) ${ }^{19}$ tem-se debruçado sobre a prevalência do fenómeno em médicos internos da especialidade de psiquiatria de adultos e da infância e da adolescência na realidade europeia.

Partindo dos resultados do BoSs em Portugal, o presente trabalho tem por objetivo analisar as características do comportamento suicidário nos médicos internos das especialidades de psiquiatria de adultos e psiquiatria da infância e da adolescência.

\section{MATERIAL E MÉTODOS}

Este estudo apresenta uma metodologia transversal, observacional e quantitativa. Para a elaboração deste trabalho foram usados os resultados obtidos na avaliação demográfica geral e as respostas às questões do Suicide Ideation and Behaviour Questionnaire (SIBQ), ${ }^{20}$ um dos questionários estruturados que foram utilizados no estudo BoSS.

O SIBQ é um instrumento em forma de questionário, constituído por 14 itens. As primeiras nove respostas, no formato sim/não, avaliam a parte dimensional da ideação suicida, desde a forma passiva (questões 1 e 2) ao comportamento suicidário (questões 3 a 8) e tentativas de suicídio (questão 9). A décima questão avalia o desejo de cometer suicídio numa escala de Likert. As duas últimas questões estão no formato 'sim/não' e exploram a história familiar de tentativa e consumação de suicídio.

O instrumento, ainda não validado para a população portuguesa, foi traduzido por uma das autoras para português utilizando a metodologia denominada back translation. Após tradução o questionário foi enviado por e-mail a todos os 159 médicos internos das especialidades de psiquiatria de adultos e de psiquiatria da infância e da adolescência em Portugal, que no ano de 2009 constavam na base de dados da Associação Portuguesa de Internos de Psiquiatria (APIP). As respostas foram recolhidas de forma automática, anónima e encriptada, numa base de dados central europeia.

\section{RESULTADOS}

Do total de 159 internos, 62 responderam ao questionário (40,3\%). Dois terços eram do sexo feminino e um terço era do sexo masculino (ratio aproximado de 2:1). A média de idade foi de 29 anos (com desvio padrão de 2,5 anos). Aproximadamente $43 \%$ dos internos eram solteiros

Tabela 1 - Dados socio-demográficos $(n=62)$

\begin{tabular}{|c|c|c|c|}
\hline & & $\mathrm{n}$ & $\%$ \\
\hline \multirow{2}{*}{ Género } & Masculino & 20 & 32,3 \\
\hline & Feminino & 42 & 67,7 \\
\hline \multirow{4}{*}{ Estado Civil } & Casado & 16 & 25,8 \\
\hline & União de facto & 17 & 27,4 \\
\hline & Solteiro & 26 & 43,5 \\
\hline & Divorciado/Separado/Sem resposta & 3 & 3,3 \\
\hline \multirow{2}{*}{ Filhos } & Sim & 10 & 16,1 \\
\hline & Não & 52 & 83,9 \\
\hline \multirow{3}{*}{ Habitação } & Casa própria & 43 & 70,5 \\
\hline & Arrendamento & 12 & 19,7 \\
\hline & Com os pais/outro & 6 & 9,8 \\
\hline
\end{tabular}


a maioria vivia em casa própria e não tinha filhos (Tabela 1). Responderam ao SIBQ 46 internos (74,2\%). Aproximadamente $44 \%$ dos internos manifestaram ideação suicida na forma passiva e $33 \%$ na forma ativa (Tabela 2).

No que diz respeito aos antecedentes familiares, $21,7 \%$ respondeu ter familiares em primeiro grau com história de tentativas de suicídio e em $13 \%$ havia história de suicídio consumado (Tabela 3 ).

$\mathrm{Na}$ apresentação dos resultados não houve lugar a descriminação por especialidade uma vez que este estudo faz parte de um estudo internacional em que na grande maioria dos países envolvidos, as duas especialidades fazem parte de um tronco comum.

\section{DISCUSSÃO}

A ideação suicida nos internos de psiquiatria em Portugal não diferiu de forma estatisticamente significativa dos valores encontrados no BoSS study: ideação suicida passiva $43,5 \%$ vs $34,2 \%$, ideação suicida activa $32,6 \%$ vs $26,2 \%$ e tentativas de suicídio $4,3 \%$ vs $2,0 \%$ (Jovanovic $\mathrm{N}$ et al. Comunicação pessoal, "Mental Health of European Psychiatry Trainees - Results from the BoSS Study" at the $20^{\text {th }}$ Forum of the European Federation of Psychiatric Trainees, May 21-26, 2012 in Sorrento, Italy), contudo, estes resultados continuam a merecer uma análise cuidada.

Em Portugal, os trabalhos que têm sido desenvolvidos sobre a ideação suicida em internos de psiquiatria têm evidenciado potenciais factores como causas do desejo de morte nesta população. Entre esses factores encontramse a insatisfação com o internato complementar, com as condições de trabalho (Castro L, Comunicação Pessoal,
"BOSS: The International Psychiatric Trainee Burnout Study" at the Young Psychiatrists' Symposium, European Psychiatric Association Congress, Munich 2010) e os sintomas de depressão. ${ }^{9}$ Além disso, os internos de psiquiatria lidam com pacientes com sintomas depressivos e comportamento suicidário na sua prática clínica, o que pode constituir um factor de stress acrescido. ${ }^{7}$ De acordo com os resultados europeus do BoSS, os comportamentos suicidários aumentam com a carga laboral, ${ }^{9}$ o que seria outra variável a ter em conta na realidade portuguesa, dado o número crescente de procura por cuidados em serviços de psiquiatria e saúde mental.

Por outro lado, factores pessoais como a idade, o neuroticismo (traços de personalidade que incluem instabilidade, insegurança emocional, ansiedade elevada, estado permanente de preocupação, com tendência à culpabilidade e geralmente associados a sintomatologia psicossomática) e abertura à experiência parecem aumentar estes comportamentos, enquanto que a maior idade de início do internato, a partilha de valores e a extroversão podem ser factores protectores. ${ }^{9} \mathrm{~A}$ própria escolha da especialidade de psiquiatria pode estar relacionada com a presença de patologia psiquiátrica na família, ${ }^{10,11}$ o que se verificou em $21,7 \%$ dos participantes no estudo. A influência familiar, quer genética quer ambiental, pode contribuir para os comportamentos suicidários demonstrados neste e noutros estudos. Questões de teor sociológico, filosófico e religioso presentes na ideação suicida também não devem ser excluídas.

Neste estudo, as tentativas de suicídio ocorreram antes da escolha da especialidade, podendo estar relacionadas

Tabela 2 - Respostas ao SIBQ $(n=46)$

\begin{tabular}{|c|c|c|c|c|c|c|}
\hline \multicolumn{3}{|l|}{ SIBQ } & Sim & $\%$ & Não & $\%$ \\
\hline \multicolumn{3}{|c|}{ Q.1 - Ideação suicida passiva: Já alguma vez pensaste que seria melhor se morresses? * } & 20 & 43,5 & 26 & 56,5 \\
\hline \multicolumn{3}{|c|}{ Q.2 - Ideação suicida activa: Já alguma vez pensaste em cometer suicídio? ** } & 15 & 32,6 & 31 & 67,4 \\
\hline \multicolumn{3}{|c|}{ Q.3 - Já alguma vez pensaste em como poderias cometer suicídio (ex. tomando comprimidos)? ** } & 13 & 28,3 & 33 & 71,7 \\
\hline \multicolumn{3}{|l|}{ Q.4 - Já alguma vez tiveste intenção de cometer suicídio? } & 6 & 13,3 & 40 & 86,9 \\
\hline \multicolumn{3}{|c|}{ Q.5 - Já alguma vez elaboraste um plano de como cometer suicídio? } & 2 & 4,3 & 44 & 95,7 \\
\hline \multicolumn{3}{|c|}{$\begin{array}{l}\text { Q.6 - Já alguma vez realizaste acções no sentido de cometer suicídio (recolhendo comprimidos, } \\
\text { carregando uma arma, escrevendo uma nota de suicídio...)? }\end{array}$} & 3 & 6,5 & 43 & 93,5 \\
\hline \multicolumn{3}{|c|}{$\begin{array}{l}\text { Q.7 - Já alguma vez deste passos no sentido de te matar mas abortaste o acto (tu mesmo) antes } \\
\text { que acontecesse alguma coisa? }\end{array}$} & 1 & 2,2 & 45 & 97,8 \\
\hline \multicolumn{3}{|c|}{$\begin{array}{l}\text { Q.8 - Já alguma vez deste passos no sentido de te matar mas foste interrompido (por alguma coisa } \\
\text { ou alguém) antes que acontecesse alguma coisa? }\end{array}$} & 1 & 2,2 & 45 & 97,8 \\
\hline \multicolumn{3}{|l|}{ Q.9 - Já alguma vez fizeste uma tentativa de suicídio? } & 2 & 4,3 & 44 & 95,7 \\
\hline \multicolumn{7}{|c|}{${ }^{*} \mathrm{n}=4$, depois de iniciar a especialidade; ${ }^{* *} \mathrm{n}=2$, depois de iniciar a especialidade. Restantes respostas afirmativas, sempre antes de iniciar a es } \\
\hline & $\mathbf{n}$ & $\%$ & & & & \\
\hline Q.11 - Tentativas de suicídio em familiares $1^{\circ} \mathrm{grau}$ & 10 & $21,7 \%$ & & & & \\
\hline Q.13 - História de suicídio consumado na família & 6 & $13,0 \%$ & & & & \\
\hline
\end{tabular}


com os níveis de psicopatologia ansiosa e depressiva detectados em estudantes de medicina portugueses. ${ }^{6}$

Os resultados obtidos assemelham-se aos dados internacionais e indiciam que existe uma maior vulnerabilidade nos internos de psiquiatria face à população geral, contudo não nos permitem concluir que os internos de psiquiatria têm um nível superior de comportamentos suicidários face aos internos de outras especialidades.

Seria muito interessante compararmos com mais detalhe os resultados em Portugal com os verificados noutros países, mas tal não é possível enquanto os resultados finais do projecto BoSS não forem divulgados a nível internacional. No entanto, sabemos que as primeiras publicações estão eminentes e muito em breve poderemos enquadrar a situação portuguesa no panorama geral.

No que diz respeito às limitações do presente trabalho, destaca-se o facto do questionário ser online e, apesar de favorecer o anonimato, as respostas podem estar condicionadas pela facilidade com que os indivíduos lidam com a internet.

As respostas ao SIBQ (e o facto deste questionário ter sido respondido somente por parte da amostra total de internos) pode reflectir predominantemente a história dos médicos internos mais sensíveis a esta temática, quer pela história pessoal como familiar. Admite-se, também, que a não validação da tradução do SIBQ para a população portuguesa possa ter prejudicado (ainda que de uma forma pouco pronunciada) a interpretação das perguntas por parte dos participantes.

\section{CONCLUSÃO}

Os comportamentos suicidários encontrados podem estar associados a factores específicos a que esta população está exposta, nomeadamente a insatisfação e stress laborais que tantas vezes levam à síndrome de burnout. Por outro lado poderão estar presentes antes da inserção na vida profissional, ou seja, durante o curso de medicina. Nesse sentido, será necessário aprofundar a investigação no âmbito deste tema, para melhor se poder compreender este fenómeno, respectivas causas e potenciais modificadores.

Este estudo teve o intuito de promover a reflexão sobre o comportamento suicidário nos médicos internos de psiquiatria em Portugal, sendo necessário mais estudos para que haja um maior aprofundamento de tais questões acerca das particularidades da prática clínica na área da psiquiatria, em particular no que diz respeito à saúde mental destes profissionais. A colaboração científica com um grupo internacional permitirá mais comparações epidemiológicas e o desenvolvimento de estratégias de redes de apoio a estes profissionais, com potencial profilático, priorizando grupos mais vulneráveis.

\section{CONTRIBUTOS DOS AUTORES}

João Gama Marques foi co-coordenador do estudo BoSS em Portugal e elaborou o primeiro manuscrito; Alice Roberto foi responsável pela revisão bibliográfica, tratamento estatístico dos dados nacionais e revisão e submissão do artigo; Cátia Guerra e Mariana Pinto da Costa foram responsáveis pela revisão bibliográfica tendo apoiado a revisão do artigo; Anja Podlesek fez o tratamento estatístico de dados a nível internacional; Nikolina Jovanovic e Julian Beezold foram os coordenadores internacionais responsáveis pela elaboração do estudo; Ana Moscoso foi co-coordenadora nacional do estudo BoSS em Portugal, realizou a tradução para português dos diferentes questionários, foi responsável pela revisão estatística dos dados nacionais e pela revisão do artigo.

\section{OBSERVAÇÕES}

Dados parciais do trabalho foram apresentados no VIII Congresso Nacional de Psiquiatria, Coimbra (2011) e no IX Congresso Nacional de Psiquiatria, Porto (2012), ambos em formato comunicação oral.

\section{PROTECÇÃO DE PESSOAS E ANIMAIS}

Os autores declaram que os procedimentos seguidos estavam de acordo com os regulamentos estabelecidos pelos responsáveis da Comissão de Investigação Clínica e Ética e de acordo com a Declaração de Helsínquia da Associação Médica Mundial.

\section{CONFIDENCIALIDADE DOS DADOS}

Os autores declaram ter seguido os protocolos do seu centro de trabalho acerca da publicação de dados.

\section{CONFLITOS DE INTERESSE}

Os autores declaram que não houve conflitos de interesse na realização deste trabalho.

\section{FONTES DE FINANCIAMENTO}

Este trabalho não recebeu qualquer contribuição, subsídio ou bolsa.

\section{REFERÊNCIAS}

1. Ionline (2010). Portugal é o país da Europa com mais doentes mentais. [Consultado 2013 Mar 10]. Disponível em http://www1.ionline.pt conteudo/52456-portugal-e-o-pais-da-europa-com-mais-doentesmentais.

2. INE (2008). Suicídio é a causa de morte não-natural mais comum no país. [Consultado em 2013 Mar 10]. Disponível em http://www.publico. pt/Sociedade/suicidio-e-a-causa-de-morte-naonatural-mais-comumno-pais 1427087.

3. Pordata (2012). Óbitos de residentes em Portugal por algumas causas de morte nos Municípios. [Consultado em 2013 Mar 10]. Disponível 
specialists among 18,730 consecutive physician deaths during a fiveyear period, 1967-72. J Clin Psychiatry. 1980;41:261-3.

8. Bergman J. The suicide rate among psychiatrists revisited. Suicide Life Threat Behav. 1979;9:219-26.

9. Maslach C, Jackson SE, Leiter MP. Maslach Burnout Inventory Manual. $3^{\text {rd }}$ ed. Palo Alto: Consulting Psychologists Press, Inc.; 1996.

10. Dyrbye LN, Thomas MR, Massie FS, Power DV, Eacker A, Harper W et al. Burnout and suicidal ideation among U.S. medical students. Ann Intern Med. 2008;149:334-41.

11. Roberto A, Almeida A. A saúde mental de estudantes de medicina: estudo exploratório na Universidade da Beira Interior. Acta Med Port 2011;24: 279-86.

12. Chemtob CM, Hamada RS, Bauer G, Kinney B, Torigoe RY. Patients suicides: frequency and impact on psychiatrists. Am J Psychiatry. 1988;145:224-8.

13. Hawton K, Clements A, Sakarovitch C, Simkin S, Deeks JJ. Suicide in doctors: a study of risk according to gender, seniority and specialty in medical practitioners in England and Wales, 1979-1995. J Epidemiol Community Health. 2001;55,296-300.

14. Dyrbye LN, Thomas MR, Massie FS, Power DV, Eacker A, Harper W et al. Burnout and suicidal ideation among U.S. medical students. Ann
Intern Med. 2008:149:334-41.

15. Peisah C, Latif E, Wilhelm K, Williams B. Secrets to psychological success: why older doctors might have lower psychological distress and Burnout than younger doctors. Aging Ment Health. 2009;13:300-7.

16. Shanafelt TD, Boone S, Tan L, Dyrbye LN, Sotile W, Satele D, et al Burnout and satisfaction with work-life balance among US physicians relative to the general US population. Arch Intern Med. 2012;172:137785

17. Medscape (2015). Physician burnout: it just keeps getting worse [Consultado 2015 Mar 10]. Disponível em: http://www.medscape.com/ viewarticle/838437.

18. Medscape (2015). Physician suicide 101: secrets, lies, and solutions [Consultado 2015 Mar 10]. Disponível em: http://www.medscape.com/ viewarticle/834434.

19. Jovanovic N, Beezhold J, Andlauer O, Kuzman MR, Podlesek A Hanon C, et al. Burnout among psychiatry residents: The International Psychiatry Resident/Trainee Burnout Syndrome Study (BoSS). Die Psychiatrie. 2009,6:75-79.

20. Marusic A, Roskar S, Sveticic J, Zorko M. Assessment and measurement of suicide risk. In: Suicide prevention in developing countries. Amresh KS editor. London: Gaskell; 2007. 
João GAMA MARQUES, Alice ROBERTO, Cátia GUERRA, Mariana PINTO DA COSTA, Anja PODLESEK, Julian BEEZHOLD, Nikolina JOVANOVIC, Ana MOSCOSO

\section{Comportamento Suicidário nos Internos de Psiquiatria em Portugal: Comparação com a Realidade Europeia}

Acta Med Port 2015:28:608-612

Publicado pela Acta Médica Portuguesa, a Revista Científica da Ordem dos Médicos

Av. Almirante Gago Coutinho, 151

1749-084 Lisboa, Portugal.

Tel: +351218428215

E-mail: submissao@actamedicaportuguesa.com

www.actamedicaportuguesa.com

ISSN:0870-399X | e-ISSN: 1646-0758

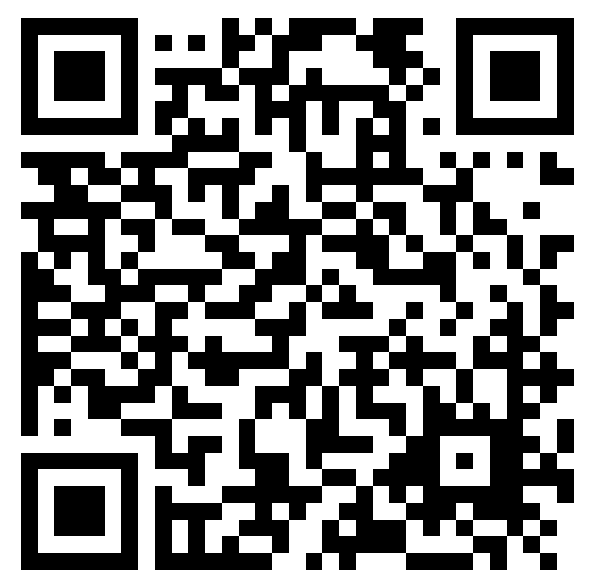

